\title{
Praxis der Wirtschaftsinformatik
}

\section{Heft 287}

Sonderdruck

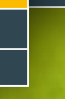

Uwe Pilgram · Alexander Vogedes

\section{Ein Geschäftssystem für ICT-Dienstleister nach industriellen Maßstäben}

Elektronischer Sonderdruck 


\section{Elektronischer Sonderdruck}

aus:

HMD - Praxis der Wirtschaftsinformatik

Diana Ingenhoff · Andreas Meier (Hrsg.)

Social Media

49. Jahrgang - Heft 287 - Oktober 2012

Seiten 103-112

(C) dpunkt.verlag $\mathrm{GmbH}$

ISSN 1436-3011

ISBN 978-3-89864-805-9 
Uwe Pilgram, Alexander Vogedes

\section{Ein Geschäftssystem für ICT-Dienstleister nach industriellen Maßstäben}

Dienstleistungen im Bereich ICT (Information and Communication Technology) sind ein zentraler Bestandteil der Unterstützung von Geschäftsprozessen bis hin zu deren automatisierter Abwicklung. Die Akzeptanz der ICT-Organisationen entspricht jedoch häufig nicht der Bedeutung ihrer Dienstleistungen. Schwachstellenanalysen zeigen, dass wesentliche Ursachen der fehlenden Akzeptanz sowie der Mängel im Management der Leistungsbeziehung zwischen dem ICT-Dienstleister und seinen Kunden liegen. Deshalb wurde das hier vorgestellte Geschäftssystem indICT für ICT-Dienstleister so gestaltet, dass es auf Planung und Realisierung von nutzenorientierten Leistungszusagen für ICT-Dienstleistungen ausgerichtet ist. Das Geschäftssystem wurde erfolgreich als Prototyp implementiert und in einem zweiten Schritt pilotiert. Besonders im Cloud Computing ist ein am Kundennutzen ausgerichtetes Geschäftssystem notwendig.

\section{Inhaltsübersicht}

1 ICT-Bereiche haben Handlungsbedarf

2 Das Geschäftssystem indICT

2.1 Leistungsbeziehung mit den Fachbereichen

2.2 Management von Bereitstellung und Leistungserstellung

2.3 Rollen und Verantwortlichkeiten

2.4 Das Modell der ICT-Prozesse

2.5 Die IT-Unterstützung des

Geschäftssystems indICT

3 indICT schafft ein neues Niveau im ICT-Management

4 Literatur

\section{ICT-Bereiche haben Handlungsbedarf}

Ein Konsortium deutscher und Schweizer Firmen hat vor mehreren Jahren zusammen mit der Universität St. Gallen ein "Competence Center Industrialisierung des Informationsmanagements (CC IIM) « geschaffen und forscht dort über eine grundlegende Weiterentwicklung des ICT-Managements. Dabei wurden die Ursachen für die Schwierigkeiten der ICT-Bereiche untersucht und ein neues Managementmodell für Geschäftssysteme von ICT-Dienstleistern entwickelt (vgl. u.a. [Brocke 2011], [Ebert 2009], [Vogedes 2011]). Dieses Modell wurde in mehreren Praxisprojekten auf seine Machbarkeit positiv überprüft. Eines der Projekte trug die interne Projektbezeichnung indICT (industrialisiertes ICT-Management), was auch als Bezeichnung des vorgestellten Konzepts dient.

Das Modell ist auch für externe ICT-Dienstleister jeder Art und für die Leistungsbeziehung zwischen ICT-Dienstleistern anwendbar.

Die Untersuchungen der Prozesse einer Reihe von ICT-Bereichen des zuvor erwähnten "Competence Center Industrialisierung des Informationsmanagements (CC IIM) « zeigen interessante Ergebnisse: Das Management der ICTBereiche ist stark auf Projekte zur Unterstützung des "Change the Business" ausgerichtet. Das Management der aktuellen ICT-Dienstleistungen für das »Run the Business" ist dagegen häufig schwach ausgeprägt. Hier jedoch entsteht der Nutzen der ICT-Dienstleistungen und liegt der Fokus der Fachbereiche.

Die Analysen ergaben folgendes Bild, aus dem die Anforderungen an das neue Managementsystem ermittelt werden: 
- Der Nutzen für die Geschäftsprozesse durch die im "Run the Business" eingesetzten ICTDienstleistungen ist kaum bekannt. Es gab praktisch keine Nutzenvereinbarungen der ICT-Bereiche mit den Fachbereichen.

- Die Leistungszusagen (SLAs) des ICT-Bereichs sind nicht auf die Geschäftsprozesse ausgerichtet und beschreiben stattdessen fast immer Leistungen aus Sicht der ICT-Bereiche. Sie sind in IT-Sprache dokumentiert, den "Betroffenen " kaum bekannt und stammen aus unterschiedlichen Quellen, häufig auch aus Telefonaten.

- SLAs machen keine Qualitätszusagen, die den Nutzen bei den Fachbereichen absichern.

- Die Prozesse der Bereitstellung und des Betriebs sind außengesteuert und passiv. Incident Management und Problemmanagement sind deshalb häufig die einzigen ITILProzesse, die wirklich als Betriebsprozesse umgesetzt sind.

- Die Organisation der ICT-Bereiche ist nach der Technologie der Betriebsmittel aufgestellt (Desktop, Netz, Rechenzentrum, Anwendungen) und nicht nach ICT-Dienstleistungen.

- Es gibt keine Auftragsbearbeitung für Bereitstellung und Leistungserbringung.

- Eine Standardisierung und Wiederverwendung von Betriebsmitteln ist selten.

- Eine leistungsfähige Kostenrechnung, die eine Kostensteuerung erlaubt, fehlt häufig.

- Management des Leerstands und der Auslastung von Betriebsmitteln sowie der "Cost of Poor Quality“ gibt es kaum. Hier liegen erhebliche Kostensenkungspotenziale.

\section{Das Geschäftssystem indICT}

Mit dem Modell des CC IIM wird nachgewiesen [Zarnekow et al. 2005], dass die geschilderten Probleme durch die Nutzung von Lösungen aus der Sachgüterindustrie behoben werden können. In der Folge hat ein Mitglied des CC IIM, T-Systems International, zusammen mit den Universitäten St. Gallen und Magdeburg 2008 das
Modell mit dem Aufbau eines Prototyps eines Geschäftssystems für SaaS-Dienstleistungen (SaaS - Software as a Service) erprobt und darüber berichtet [BITKOM 2009; FH Brandenburg 2010].

\subsection{Leistungsbeziehung mit den Fachbereichen}

Die Beziehung zu den Fachbereichen ist so gestaltet, dass sämtliche aktuell zu liefernden ICTDienstleistungen als ein Portfolio von Dienstleistungsprodukten eindeutig nach Funktionen, Qualität, Preisen und Liefermengen beschrieben sind. Diese gegenseitigen Zusagen sind allen Stakeholdern bekannt und verständlich sowie jederzeit einsehbar. Die Leistungszusagen stehen in einer nachvollziehbaren Beziehung zum erwarteten Nutzen in den Geschäftsprozessen.

Im Planungsprozess werden neue ICTDienstleistungen definiert, entwickelt oder bestehende angepasst. Für das "Run the Business « erfolgt in den operativen Prozessen der Abruf und die Verwaltung der im Portfolio enthaltenen ICT-Dienstleistungen für den konkreten Einsatz in den Geschäftsprozessen.

Die gegenseitigen Leistungszusagen werden nach folgenden Regeln definiert und dokumentiert:

\section{Nutzen- und Anwenderorientierung}

- Für jede ICT-Dienstleistung gibt es genau eine einzige verbindliche Beschreibung von Leistungszusagen: die standardisiert aufgebaute Service Description.

- Leistungszusagen enthalten Zusagen zu Funktionen, Mengen, Qualität und Kosten sowie zum Nutzen in den Geschäftsprozessen.

- Die Qualitätsmerkmale der Leistungszusagen haben einen sachlich nachvollziehbaren Zusammenhang mit dem Nutzen der ICTDienstleistung für einen Geschäftsprozess.

- Im Qualitätsmanagement werden Störungen der zugesagten Leistungsqualität (z. B. zugesagte Bereitstellungszeiten oder Antwortzei- 
ten) vom Dienstleister durch Messung erkannt und direkt behoben (Six Sigma), möglichst bevor der Anwender eine Störung bemerkt.

- Die Anwenderzufriedenheit ist als ein Indikator für den Nutzen ein wichtiges Qualitätsmerkmal.

\section{Produktorientierung und Rahmenverträge}

- ICT-Dienstleistungen werden zu genau abgegrenzten ICT-Produkten gruppiert, die Leistungszusagen enthalten.

- Der Bedarf der Fachbereiche wird als Ergebnis des Planungsprozesses in Leistungszusagen von ICT-Produkten umgesetzt. Dabei plant der ICT-Bereich selbstständig im Rahmen seiner Servicestrategie.

- ICT-Produkte beschreiben "was wird geliefert" und nicht »wie arbeitet der ICT-Bereich". Es gilt die Regel: Gleiche Leistungszusagen sind identisch formuliert.

- Qualitätsmerkmale sichern den Nutzen einer ICT-Dienstleistung.

- Die Fachbereiche bestellen selbstständig aus einem vereinbarten Katalog von ICT-Produkten den tatsächlichen Bedarf an ICT-Dienstleistungen.

- Die zur Erfüllung der Leistungszusagen notwendigen internen Spezifikationen des ICTBereichs für Bereitstellung und Betrieb sind als ICT-Fertigungsprodukte dokumentiert.

Zusammengefasst führen diese Anforderungen zu einem grundlegend veränderten anwenderorientierten Servicemanagement und einer deutlich verbesserten Auftragsbearbeitung.

\subsection{Management von Bereitstellung und Leistungserstellung}

Die Aufgabe des Managements von Bereitstellung und Leistungserstellung besteht darin, die Leistungszusagen an die Fachbereiche zu erfüllen. Dazu werden im Rahmen der Planungsprozesse die Lösungen für die Anforderungen gestaltet und Entscheidungen über Art und Einsatz sämtlicher dafür erforderlichen Betriebsmittel getroffen. Dazu gehört auch Anwendungssoftware. Bei der Planung der Betriebsmittel stehen Auslastung und Standardisierung im Vordergrund. Die operativen Prozesse erledigen die Kapazitätsplanung für Betriebsmittel, das Sourcing von Vorleistungen und Technologie, die Bereitstellung der Betriebsmittel, den Betrieb als eigentliche Leistungserbringung, das Change Management, den Service-Desk und das Incident Management.

\section{Planung von Betriebsmitteln}

Unter Betriebsmitteln werden installierte und betriebsbereite Infrastruktur, Hardware und Anwendungen und sonstige Technologie, insbesondere Netze, verstanden. Die Planung von Betriebsmitteln unterscheidet sich deutlich von der Sachgüterindustrie, weil die Inanspruchnahme der Kapazitäten von Betriebsmitteln nicht in der für ERP (Enterprise Resource Planning) üblichen Form nur von Absatzmengen abgeleitet werden kann. Hier müssen zusätzlich Methoden (Schätzverfahren oder Ableitung aus Monitoring-Werkzeugen) zur Erstellung von Bedarfsprofilen/Lastprofilen genutzt und vor allem kurzfristige und gleichzeitig kostenwirksame Anpassungsprozesse eingesetzt werden (vgl. [Pinnow 2009]).

Ungewohnt ist auch die Vorstellung, dass persönliche Arbeitsmittel von Anwendern, z. B. ein Desktop, ein Laptop oder Tablet, Betriebsmittel sind. Entscheidend ist nur, dass es zur Erfüllung der Leistungszusagen erforderlich ist. Nicht entscheidend ist, wem ein Betriebsmittel gehört und wer es beschafft und finanziert.

\section{Management der Leistungserbringung}

Im Management der Leistungserbringung werden die erforderlichen Betriebsmittel bereitgestellt, die ICT-Dienstleistung wird "erzeugt" und den Anwendern der Fachbereiche "ausgeliefert«. Alle anderen operativen und planerischen Aufgaben haben an den Voraussetzungen für die Leistungserbringung gearbeitet. Hier wird jetzt Effizienz und Qualität sichtbar 
und messbar, indem durch den Leistungserbringer über die Einhaltung der Leistungszusagen berichtet wird. Entsprechend müssen die Prozesse gestaltet sein. Die Betreuung der Anwender der Fachbereiche durch ein Delivery Management stellt sicher, dass einfache Anpassungen an den Bedarf der Anwender umgesetzt werden und die Anwender mit den erbrachten Dienstleistungen zufrieden sind.

\section{Anwender- und Output-Orientierung}

Die Fachbereiche erwarten vollständige, den Leistungszusagen entsprechende ICT-Dienstleistungen. Da bei ICT-Dienstleistungen, wie bei allen Dienstleistungen, die Leistung erst erzeugt wird, wenn sie vom Anwender tatsächlich genutzt wird, ist es sinnvoll, dem Anwender und den Fachbereichen die Erfüllung der Leistungszusagen permanent nachzuweisen. Dies bedeutet, dass der ICT-Bereich aktuelle Messwerte der vereinbarten Qualitätsmerkmale bereithält und diese sofort zur Verfügung stehen, wenn Anwender oder Fachbereich dies möchten.

\section{Kostenrechnung und Kostensteuerung}

Ein wesentlicher Bestandteil des neuen Geschäftssystems ist eine Kostenrechnung, die die Effizienz und Qualität der Leistungserbringung abbildet und so eine wirkungsvolle Kostensteuerung ermöglicht. Dabei werden zwei Kostenkomponenten, die Kosten des Leerstands von Betriebsmitteln und die "Cost of Poor Quality", erfasst und den Verantwortlichen zugeordnet. "Cost of Poor Quality" ist ein Begriff aus dem Total Quality Management (TOM) und wird in diesem Beitrag im Kontext der ICT-Leistungserbringung für die Kosten des Ressourceneinsatzes mit Qualitätsmängeln genutzt (z. B. die Kosten für ein abgebrochenes Backup, das wegen des Abbruchs wiederholt werden muss). Diese beiden Kostenkomponenten sind bei den komplexen Preismodellen für Technologie und Vorleistungen fast die einzigen konkreten Ansatzpunkte zu einer Kostensteuerung.

\subsection{Rollen und Verantwortlichkeiten}

Ein Rollenmodell für das Prozessmodell definiert Verantwortlichkeiten in den Prozessen und bildet Personengruppen. Dabei werden Aufbauorganisation und Hierarchien nicht berücksichtigt. Die Strukturierung ist identisch zum Prozessmodell.

Die Rollen in den Planungsprozessen sind:

- Account Management zur Ermittlung der Kundenwünsche, Erläuterung des Produktportfolios und Abschluss von Rahmenverträgen

- Produktmanagement mit Verantwortung zur Planung und Definition eines Produktportfolios nach Kunden- und Marktanforderungen

- Produktgestaltung (Product Engineering) mit der Verantwortung zur Planung und Entwicklung von Lösungskomponenten (z.B. Anwendungssoftware)

- Produktionsgestaltung (Production Engineering) mit Verantwortung für Architektur und Auswahl von Betriebsmitteln und Technologie sowie für das Management von Inbetriebnahmeprojekten

Die Rollen in den operativen Prozessen sind:

- Beschaffungsplanung und Beschaffung mit Verantwortung für Lieferantenmanagement und Einkauf von Vorleistungen und aller Betriebsmittel

- Betriebsmittelmanagement mit Verantwortung für Kapazitätsplanung, Installation, Bereitstellung und Wartung aller Betriebsmittel

- Management der Leistungserbringung (Fulfillment) mit Verantwortung für Produktion und Service-Desk einschließlich Incident Management

- Delivery Management als Management der Anwenderbeziehung mit Verantwortung für Rollouts, Ausnahmeregelungen, Problemmanagement und die Anwenderzufriedenheit. Auch Leistungszusagen zum Nutzen werden hier verantwortet. 


\subsection{Das Modell der ICT-Prozesse}

Es werden Prozessmodelle aus der Sachgüterindustrie adaptiert. Dabei sind einige Besonderheiten von ICT-Dienstleistungen zu beachten, die auch für ICT-Dienstleistungen gelten, die dem Cloud Computing zugeordnet sind. Diese sind:

- die Abbildung einer dauerhaften Leistungsbeziehung ohne Auftragscharakter mit einem Lifecycle,

- die Abbildung einer nicht lagerfähigen Dienstleistung, die erst entsteht, wenn der Fachbereich sie abruft, als ICT-Produkt,

- die Abbildung eines Fabrikmanagements mit von Kundenaufträgen stark abhängigen Kapazitätsanpassungen.

Es werden zwei Gruppen von Prozessen unterschieden:

- "Change the Business (CTB)« schafft die Rahmenbedingungen für zukünftiges Geschäft des ICT-Bereichs und dient somit in erster Linie seiner Weiterentwicklung und seines Leistungsangebots.

- "Run the Business (RTB)« erfüllt die aktuellen Ziele, dient also in erster Linie der Erfüllung eingegangener Verpflichtungen und der Erreichung vereinbarter Ziele (Nutzen in Geschäftsprozessen, Anwenderzufriedenheit, Umsatz, EBIT (Earnings Before Interest and Taxes) etc.).

Weitere Prozesse wie Beschaffung, Finanzen und Controlling sind natürlich Bestandteil der Definitionen des neuen Geschäftssystems. Sie sind den heute üblichen Implementierungen ähnlich, sodass dies hier nicht weiter behandelt wird.

\section{Die Planungsprozesse}

Die CTB-Prozesse sind:

- Vertrieb und/oder Account Management

- Produktmanagement
- Produktgestaltung (Product Engineering) mit der Lösungsentwicklung

- Produktionsgestaltung (Production Engineering)

Die Planungsprozesse sind geradlinig gestaltet. Das Produktmanagement nimmt vom Markt oder vom Vertrieb konkrete Anforderungen entgegen und entwickelt mithilfe der Produktgestaltung und der Produktionsgestaltung eine Lösung für die Anforderung, also ein neues ICTProdukt mit einer Kalkulation der Kosten.

Jedem neuen ICT-Produkt ist ein ICT-Fertigungsprodukt zugeordnet. Es beschreibt mit Stücklisten und Arbeitsplänen die Bereitstellung und die Leistungserstellung des ICT-Produkts. Dabei wird auf modulare, standardisierte Vorleistungen zurückgegriffen, die als Materialstammdaten vorhanden sind. Ein hoher Grad der Wiederverwendung solch standardisierter Vorleistungen in beiden Funktionen ist ein wichtiges Ziel dieser Rollen. Das Produktmanagement verwendet selbst "CommitmentBausteine" als Elemente der Service Descriptions, z.B. standardisierte Qualitätsmerkmale.

Die Ergebnisse des Planungsprozesses sind in erster Linie die inhaltlich und formal standardisierten Service Descriptions der ICT-Produkte. Die Service Descriptions enthalten auch Preise, Bestellinformationen und exakte Qualitätsmerkmale. Aus innen werden dann alle weiteren Daten und Informationen für Vertrieb und Produktion abgeleitet. Die Service Descriptions werden unter der Produktnummer des ICT-Produkts "versioniert" und können von den Anwendern jederzeit eingesehen werden. Diese Informationen werden strukturiert aufbereitet und veröffentlicht.

Die weiteren Ergebnisse des Planungsprozesses sind der ICT-Produktkatalog als ein Verzeichnis der bestellbaren ICT-Produkte und die ICT-Fertigungsprodukte mit den Bibliotheken der standardisierten Vorleistungen und der Absatzplan des Produktmanagements. Letzterer ist eine Zusammenfassung der Produktions- 
mengen an ICT-Produkten, bestehend aus Abschätzung für bestehende Aufträge und Erwartungen zu neuen Aufträgen und neuen Kunden.

\section{Die operativen Prozesse}

Die RTB-Prozesse sind:

- Die Absatzwirtschaft mit den Teilprozessen Auftragsbearbeitung (Order to Cash), ExceptionHandling, Problemmanagement, der Faktura und der Einführung neuer Produkte beim Fachbereich sowie dem Management der Anwenderzufriedenheit

- Die Produktionswirtschaft mit den Teilprozessen Leistungserstellung mit Service-Desk und Incident Management sowie Betriebsmittelmanagement mit Bereitstellung, Wartung von leistungsbereiten Betriebsmitteln und Change Management

Die Absatzwirtschaft bearbeitet Bestellungen unter Berücksichtigung der Kundenbestände an Dienstleistungsprodukten bis zum fehlerfreien Kundenauftrag, legt eine neue Bestandsposition mit den Daten des bestellten ICT-Produkts an und übergibt den Kundenauftrag an die Produktionswirtschaft. Diese erstellt daraus einen Fertigungsauftrag, der dann die weiteren Prozesse der Produktionswirtschaft steuert und Rückmeldungen entgegennimmt. Ein technisches Auftragsmanagement kann entfallen.

Ein wichtiger Grundsatz ist, dass alle auszuführenden Aktivitäten der Produktionswirtschaft von Aufträgen ausgelöst werden, gegen die dann Fertigungsrückmeldungen zu liefern sind, in denen Mengen, verwendete Kapazitäten und die Qualität des Ergebnisses berichtet werden. Diese "geschlossenen " Prozesse bilden die Grundlage für die Nachkalkulation, die Berichterstattung dem Kunden gegenüber, die Kapazitätsplanung und das Qualitätsmanagement.

\section{Die Prozesse der Absatzwirtschaft}

Aufträge des Kunden werden grundsätzlich von Mitarbeitern des Kunden, den "Anwendern" selbst oder einem Beauftragten aus einem kun- denspezifischen Bestellkatalog in einem SelfService-Portal in der Konfigurationsbeziehung zu den vorhandenen Beständen an ICT-Produkten erteilt.

Dazu ist eine versionierte Bestandsführung aller bestellten ICT-Produkte über den gesamten Lifecycle hinweg, vom Auftrag über die Auftragsbearbeitung, die Bereitstellung und die Leistungserstellungsphase bis zum Rückbau nach einer Kündigung, erforderlich. Damit wird eine lückenlose Dokumentation der aktuellen Leistungsbeziehung erreicht. Dies ist für Dauerleistungsverhältnisse und Einmalleistungen gleichermaßen wichtig. Dieser Bestand enthält alle Daten aus dem Auftrag und die Istdaten von Qualitätsmerkmalen und Mengen sowie anderen bestandspflichtigen Informationen (Datum/Uhrzeiten, Besteller, Links zu den Service Descriptions etc.). Die Istdaten werden über das ICT-Fertigungsprodukt aus Fertigungsrückmeldungen in das ICT-Produkt übertragen. Dort kann der Kunde diese Informationen abrufen und auswerten.

Zur Abbildung der Konfigurationsbeziehung ist der Bestellkatalog, aus dem der Anwender seinen Auftrag erteilt, »bestandssensitiv«. Der Anwender wählt im Bestand eine Position aus und der Katalog zeigt, welche ICTProdukte im Kontext dieser Bestandsposition möglich sind. So sind Aufträge immer zu $100 \%$ konsistent und kompatibel zum Bestand, was eine Auftragsklärung unnötig macht. Gibt es Fragen zur Gestaltung der Auftragsabwicklung, z.B. die Methoden zur Übernahme von Kundendaten, werden sie in der Bereitstellung als Arbeitsplanposition in einer "Projektwerkstatt" geklärt.

In den weiteren Teilprozessen werden in der Absatzwirtschaft nach Ende der Bereitstellung Lieferscheine an den Besteller geschickt, um die Servicebereitschaft des bestellten ICT-Produkts anzukündigen. Mithilfe der Istmengen und eines in den Bestandspositionen hinterlegten Fakturamodells wird dann der Order-to-CashProzess geschlossen. Durch die in den Bestands- 
positionen vorhandenen Istdaten kann der Anwender einen Status seiner Bestandspositionen einsehen.

Die Absatzwirtschaft erzeugt auch den Kundenauftrag für die Produktionswirtschaft. Dort erstellt er einen Fertigungsauftrag und durch Verwendung von Variantenstücklisten ein spezifisches ICT-Fertigungsprodukt.

\section{Die Prozesse der Produktionswirtschaft}

Aus dem Fertigungsauftrag wird nach Ermittlung der notwendigen Betriebsmittelkapazität ein Fertigungsauftrag "Bereitstellung " erzeugt und nach Rückmeldung des Endes der Bereitstellung der Fertigungsauftrag zur "Leistungserstellung" angelegt. Die Daten aus der Bestellung bleiben in beiden Aufträgen verfügbar. Der Fertigungsauftrag zur "Leistungserstellung" ist wegen der Notwendigkeit, Dauerleistungsverhältnisse in der Produktion zu beauftragen, als ein Objekt des "Plant Maintenance implementiert, das wiederkehrende Tätigkeiten steuern kann.

Grundsätzlich gilt, dass die Bereitstellungsprozesse (auch Rückbau) materialwirtschaftliche Prozesse eines Fabrikmanagements sind. Der Bedarf an Betriebsmittelkapazität ist bei den Vorleistungen in Form von Bedarfsprofilen geplant worden. Bedarfsprofile stellen den Betriebsmittelbedarf der Vorleistungen im Zeitverlauf, z. B. während eines Monats, dar. Diese Daten werden zusammen mit der Auslastung der in Verwendung befindlichen Betriebsmittel genutzt, um den veränderten Bedarf an Betriebsmitteln zu berechnen und daraus die Stücklisten und Arbeitspläne zur Abwicklung der Bereitstellung zu ermitteln. Die in den Arbeitsplänen definierten Aktivitäten können dabei von Automaten ausgeführt werden (z.B. Anlagen von Accounts oder Shares oder Ausführen von SAP-Transporten) oder von ICT-Werkstätten (z.B. Aufbau/Abbau von Servern) oder aber von Dritten. In allen Fällen wird nach Erledigung einer Aktivität eine Rückmeldung über Ressourcenverbrauch und Erfolg der Aktivität gegeben.
Durch die Möglichkeit, Aktivitäten zu sequenzieren, wird eine Art Bereitstellungsworkflow möglich. Weiterhin werden durch die Überwachung offener Aktivitäten durch die SAP Business Suite neue Möglichkeiten der Präzision in der Bereitstellung geschaffen. Die Fertigungsrückmeldungen können bis zur Bestandsposition eines ICT-Produkts weitergeleitet werden. Damit wird dem Besteller die Möglichkeit gegeben, den Bereitstellungsprozess zu verfolgen.

Es wird ohne Weiteres deutlich, dass diese Verfahren besonders effizient sind, wenn die Anpassung von Kapazitäten in der Bereitstellung automatisiert ausgeführt werden kann. Dies wird durch den Einsatz von Virtualisierungstechniken erreicht, die generell die Partition oder Aggregation von physischen Betriebsmitteln ermöglichen. Umgekehrt können virtualisierte Betriebsmittel erst durch diese Form der automatisierten Bereitstellung einen optimalen Beitrag zur Kapazitätsoptimierung leisten (vgl. [Osterburg 2009]).

Nach Rückmeldung des erfolgreichen Abschlusses der Bereitstellung generiert der Fertigungsauftrag die notwendigen Objekte zur Beschreibung der Betriebsprozesse zur Leistungserstellung.

Der Fertigungsauftrag zur "Leistungserstellung" ist in erster Linie ein Sammler für Kapazitätsverbrauch und Rückmeldungen zur Erfüllung von Qualitätsmerkmalen. Eine weitere Aufgabe besteht darin, seinerseits Fertigungsaufträge für wiederkehrende Tätigkeiten, erneut mit Stücklisten und Arbeitsplänen, nach Planung auszuführen. Solche wiederkehrenden Tätigkeiten können z. B. das Starten und Stoppen von Servern oder das Ausführen von Backups sein. Auch hier können diese Tätigkeiten wieder von Automaten oder ICT-Werkstätten oder Dritten ausgeführt werden. Diese wiederkehrenden Tätigkeiten werden ebenfalls mit Qualitätsdaten zurückgemeldet und im ICT-Fertigungsprodukt überwacht. Entscheidend ist hier insgesamt, dass der geplante Kapazitätsbedarf und der tatsächliche Kapazitätsverbrauch 
ausgewertet und diese Informationen für taktische Anpassungen verwendet werden. Auch hier gilt wieder, dass die Automatisierung durch geeignete Technologie der Betriebsmittel möglich gemacht wird und umgekehrt. Die Einbindung und Nutzung der operativen ITILProzesse im Betrieb ist ohne Weiteres möglich und empfehlenswert.

\subsection{Die IT-Unterstützung des Geschäftssystems indICT}

Um unser neues Managementsystem in der Praxis einzusetzen, müssen die Prozesse mit einem leistungsfähigen IT-System zu einem Geschäftssystem ergänzt werden. Dieses ITSystem kann wegen der integrierten Geschäftsabwicklung nur auf einer integrierten Datenbasis aufgebaut sein. Dieses Abwicklungssystem dient der Planung, Steuerung, Abwicklung, Abrechnung und Dokumentation des Geschäfts sowie der Planung und Abrechnung der Betriebsmittel. Eine operative Steuerung oder Dokumentation von Betriebsmitteln bleibt den Systemen des IT-Servicemanagements (ITSM) überlassen. Diese Systeme führen Aufträge des Abwicklungssystems aus und melden die erreichte Qualität und den Ressourcenverbrauch an das Abwicklungssystem zurück. Das Abwicklungssystem muss folgende Funktionen implementieren:

- Unterstützung des Produktmanagements bei der Definition von ICT-Produkten unter Verwendung standardisierter Komponenten und der Erstellung von Service Descriptions sowie der Verwaltung des Angebotsportfolios und der Absatzplanung.

- Unterstützung der Absatzwirtschaft mit der Verwaltung der Stammdaten, der Vertragsdaten, der Leistungsverzeichnisse, der Bestellkataloge sowie der Bestände an ICT-Produkten über den gesamten Lifecycle, jeweils ergänzt mit Istdaten zu Mengen und Qualität.

- Außerdem sind die Funktionen Bestellung von ICT-Produkten, Erzeugen von Kundenauf- trägen aus Bestellungen, Anzeige und Auswertung der Soll- und Istbestandsdaten als Cockpit, Versand von Auftragsbestätigungen und Lieferscheinen, Faktura sowie Anzeige der Service Descriptions implementiert. Die Nutzung erfolgt über ein Self-Service-Portal mit einem "bestandssensitiven « Katalog.

- Unterstützung der Produktionswirtschaft durch die Erzeugung und Verwaltung von ICT-Fertigungsprodukten, Produktionsaufträgen und Betriebsmitteln, durch die Ermittlung des Kapazitätsbedarfs an Betriebsmitteln auf Basis der Absatzplanung, Ermittlung der zur Kapazitätsanpassung erforderlichen Aktivitäten (Bereitstellung), Verwalten der Betriebsmittel sowie Steuerung und Abrechnung der Bereitstellungs- und Betriebsaktivitäten durch Fertigungsaufträge (ersetzen Betriebshandbücher). Weiterhin wird die Ermittlung der Tarife von Vorleistungen und der Kosten der ICT-Fertigungsprodukte unterstützt. Dabei sind Stücklisten und Arbeitspläne einzusetzen.

Bei Untersuchungen zur IT-Unterstützung wurde die Anwendbarkeit von auf CRM (Customer Relationship Management)/ERP ausgerichteter Standardsoftware untersucht. Als Standardsoftware wurde die SAP Business Suite benutzt. Unsere Analysen haben gezeigt, dass die obigen Anforderungen zu mehr als $80 \%$ mit dieser Standardsoftware abgedeckt werden. Dabei ist jedoch zu berücksichtigen, dass die Schwerpunkte bei der Steuerung eines Dienstleistungsgeschäfts wesentlich anders gesetzt werden müssen als in der Sachgüterindustrie. Folgende Herausforderung müssen bewältigt werden:

- ICT-Dienstleistungen müssen als Dauerleistungsverhältnisse mit einem Lifecycle abgebildet werden.

- ICT-Dienstleistungen haben nach Auftragserteilung durch den Kunden eine Bereitstellungsphase, in der die "Fabrik umgebaut" wird und die spezifischen Betriebsprozesse 
erzeugt sowie Delta-Kapazitäten auf der Basis von Bedarfsprofilen ermittelt werden.

- Kundenaufträge sind abhängig von den bereits »in Produktion « oder »in Bereitstellung" befindlichen ICT-Dienstleistungen. Um korrekte Aufträge zu formulieren, wird statt eines üblichen Konfigurators für neue Produkte ein Konfigurator zwischen Katalog und Bestand benötigt, der "bestandssensitive" Katalog.

- Shopsysteme sind für ein Self-Service-Portal nicht geeignet. Kundenseitig ist der Status der bestehenden Leistungsbeziehung wichtiger als neue Aufträge.

- Bei den Planungsprozessen ist eine Unterstützung bei der Erstellung der Produktkataloge und Service Descriptions erforderlich.

Diese Themen sind nicht spezifisch für ICTDienstleistungen, sondern gelten generell bei „ERP für Services". Somit sind diese Anforderungen auch für ICT-Dienstleistungen des Cloud Computing zu berücksichtigen.

\section{3 indICT schafft ein neues Niveau im ICT-Management}

Die auch von T-Systems International veröffentlichten Testergebnisse [BITKOM 2009; FH Brandenburg 2010] mit einem Prototyp des ICT-Geschäftssystems zeigen, dass die zuvor dargestellten Prozesse konsistent und ausführbar sind und mit CRM/ERP-Software weitgehend unterstützt werden können.

Die bisherigen Erfahrungen belegen auch, dass durch »indICT " eine wesentlich bessere Standardisierung und damit verbunden eine einfachere Anpassung von Betriebsmittelkapazitäten sowie ein wirkungsvolles Qualitätsmanagement nach Six-Sigma-Methoden möglich werden. Es ist durch die in der Regel nach den klassischen "Produktionssäulen « aufgestellten $\mathrm{ClO}$-Bereiche nur selten möglich, Kostensenkungspotenziale bei der an die Fachseiten abgegebenen Leistung zu ermitteln, da die kalkulatorische Basis fehlt. Die zitierten Implementie- rungen haben gezeigt, dass durch eine bessere Auslastung der Betriebsmittel und durch weniger Verbrauch für die Erzeugung von »Poor Quality « die Kapazitätskosten gesenkt werden können. Mit dem indICT-Geschäftssystem können ICT-Bereiche ein neues Niveau von Kundenorientierung mit klaren am Kundennutzen ausgerichteten Leistungszusagen erreichen. Die bei der Pilotierung beteiligten Fachseiten waren wegen der großen Transparenz hinsichtlich Qualität und Kosten der neugestalteten Leistungsbeziehung begeistert (vgl. [BITKOM 2009], [FH Brandenburg 2010]). Weitere Details zu diesem Thema sind verfügbar unter [Pilgram \& Vogedes 2012].

\section{Literatur}

[BITKOM 2009] Oecking, C.; Jahnke, R.; Kienle, H.; Weber, M. (Hrsg.): Industrialisierung im Outsourcing. BITKOM-Arbeitskreis Outsourcing, 2009.

[Brocke 2011] Brocke, H.: Kundenorientierte Gestaltung und Vereinbarung standardisierter ITDienstleistungen. Universität St. Gallen, Dissertation, St. Gallen, 2011.

[Ebert 2009] Ebert, N.: Produktionsplanung und -steuerung bei IT-Dienstleistern. Universität St. Gallen, Dissertation, St. Gallen, 2009.

[FH Brandenburg 2010] Fachhochschule Brandenburg: Industrialized IT - Idee und Realität. Tagungsband zum 9. Berlin-Brandenburger SAP-Forum der Fachhochschule Brandenburg, 2010.

[Osterburg 2009] Osterburg, S.: Das Rechenzentrum als Produktionsstätte für IT-Dienstleistungen: Verfügbarkeitsmanagement in virtualisierten Rechenzentren. Otto-von-GuerickeUniversität Magdeburg, Dissertation, Magdeburg, 2009.

[Pilgram \& Vogedes 2012] Pilgram, U.; Vogedes, A.: indICT - Ein neues Geschäftssystem für ICTDienstleister, 2012, www.alexandria.unisg.ch/ Publikationen/215509.

[Pinnow 2009] Pinnow, A.: Das Rechenzentrum als Produktionsstätte für IT-Dienstleistungen - Kapazitätswirtschaft in virtualisierten Rechenzentren. Otto-von-Guericke-Universität Magdeburg, Dissertation, Magdeburg, 2009. 
[Vogedes 2011] Vogedes, A.: Ansatz eines KapaziUwe Pilgram tätsmanagements für die Erbringung von ITDienstleistungen. Universität St. Gallen, DisserUwe Pilgram ICT-Management Raiffeisenstr. 4 tation, St. Gallen, 2011.

[Zarnekow et al. 2005] Zarnekow, R.; Brenner, W.; Pilgram, U.: Integriertes Informationsmanagement. Springer-Verlag, Heidelberg, 2005.

67273 Weisenheim am Berg uwe.pilgram@t-online.de

Dr. Alexander Vogedes

IT Management Partner St. Gallen AG Oberer Graben 6 $\mathrm{CH}-9000$ St. Gallen alexander.vogedes@itmp-sg.com www.itmp-sg.com 